\title{
Operators on Pure Spinor Spaces
}

\author{
Martin Cederwall \\ Fundamental Physics, Chalmers University of Technology, SE 41296 Goteborg, Sweden \\ martin.cederwall@chalmers.se
}

\begin{abstract}
Pure spinors are relevant to the formulation of supersymmetric theories, and provide the only known way to maintain manifest maximal supersymmetry. The (non-linear) pure spinor constraint makes it nontrivial to find well defined operators on pure spinor wave functions. We discuss how such operators are defined. One application concerns covariant gauge fixing in maximally supersymmetric Yang-Mills (and string theory). Another issue is the construction of a manifestly supersymmetric action for 11-dimensional supergravity in terms of a scalar superfield. We describe some work in progress.
\end{abstract}

Keywords: extended supersymmetry, superfields, pure spinors

PACS: $11.10 \mathrm{Ef}, 11.30 \mathrm{~Pb}$

There is a close relation between supermultiplets and pure spinors. The algebra of covariant fermionic derivatives in flat superspace is generically of the form

$$
\left\{D_{\alpha}, D_{\beta}\right\}=-T_{\alpha \beta}^{c} D_{c}=-2 \gamma_{\alpha \beta}^{c} D_{c} .
$$

If a bosonic spinor $\lambda^{\alpha}$ is pure, i.e., if the vector part $\left(\lambda \gamma^{a} \lambda\right)$ of the spinor bilinear vanishes, the operator $Q=\lambda^{\alpha} D_{\alpha}$ becomes nilpotent, and may be used as a BRST operator. This is, schematically, the starting point for pure spinor superfields. (The details of course depend on the actual space-time and the amount of supersymmetry. The pure spinor constraint may need to be further specified. Eq. (1) may also contain more terms, due to super-torsion and curvature.) The cohomology of $Q$ will consist of supermultiplets, which in case of maximal supersymmetry are on-shell. The idea of manifesting maximal supersymmetry off-shell by using pure spinor superfields $\Psi(x, \theta, \lambda)$ is to find an action whose equations of motion is $Q \Psi=0$.

The fact that pure spinors had a rôle to play in maximally supersymmetric models was recognised early by Nilsson [1] and Howe [2, 3]. Pure spinor superfields were developed with the purpose of covariant quantisation of superstrings by Berkovits $[4,5,6,7]$ and the cohomological structure was independently discovered in supersymmetric field theory and supergravity, originally in the context of higher-derivative deformations $[8,9,10,11,12,13,14,15]$. The present lecture only deals with pure spinors for maximally supersymmetric field theory.

The canonical example of pure spinors is in $D=10$. There is only one nongravitational supermultiplet, namely super-Yang-Mills, so this is what we expect to 
obtain. Expanding a field $\Psi(x, \theta, \lambda)$ in powers of $\lambda$, one has

$$
\Psi(x, \theta, \lambda)=\sum_{n=0}^{\infty} \lambda^{\alpha_{1}} \ldots \lambda^{\alpha_{n}} \psi_{\alpha_{1} \ldots \alpha_{n}}(x, \theta) .
$$

The implementation of the pure spinor constraint is as an abelian gauge symmetry, where the generators $\left(\lambda \gamma^{a} \lambda\right)$ act multiplicatively. The field $\Psi$ is defined modulo the ideal generated by the constraint. A "canonical" representative of the gauge orbits is provided by superfields $\psi_{\alpha_{1} \ldots \alpha_{n}}(x, \theta)$ which, in addition to being symmetric, are completely $\gamma$ traceless, i.e., in the modules $(000 n 0)$ of the Lorentz algebra (where $\lambda^{\alpha}$ is in (00001) and $D_{\alpha}$ in (00010), the two spinor chiralities).

In order to calculate the cohomology, we start by finding the cohomology of zeromodes, $x$-independent fields. This cohomology is easy to calculate (a purely algebraic calculation), and gives information about the full cohomology. It is worth noting that the zero-mode cohomology (which clearly would have been empty for an unconstrained $\lambda$ ) may be read off from the partition function for a pure spinor. It is in one-to-one correspondence (for a concrete explanation of this fact, using the reducibility of the pure spinor constraint, see the appendix of ref. [5] and ref. [16]) with the six terms in the nominator of the partition function

$$
Z(t)=\frac{1-10 t^{2}+16 t^{3}-16 t^{5}+10 t^{6}-t^{8}}{(1-t)^{16}}=\frac{\left(1+t^{2}\right)\left(1+4 t+t^{2}\right)}{(1-t)^{11}}
$$

(This partition function only counts the dimension of the space of monomials is $\lambda$ with degree of homogeneity $p$ as the coefficient of $t^{p}$. A more refined partition function, specifying the actual Lorentz modules appearing, can of course be written down; for this I refer to ref. [16].) The zero-mode cohomology is illustrated in Table 1. There, each column represent a field in the expansion (2), and the vertical direction is the expansion in $\theta$. The columns have been shifted so that components on the same row have the same dimension, i.e., so that $Q$ acts horizontally. Since $\lambda$ carries ghost number 1 and dimension $-1 / 2$, the component field $\psi_{\alpha_{1} \ldots \alpha_{n}}$ has ghost number $g h(\Psi)-n$ and dimension $\operatorname{dim}(\Psi)+\frac{n}{2}$. It is natural to let $\operatorname{gh}(\Psi)=1$ and $\operatorname{dim}(\Psi)=0$ and take $\Psi$ to be fermionic. Then the scalar (ghost number 1, dimension 0 ) in the first column is interpreted as the Yang-Mills ghost and the vector and spinor in the second column as the fields of the super-Yang-Mills multiplet (the field $\psi_{\alpha}$ of ghost number 0 and dimension $1 / 2$ is the lowest-dimensional connection component $A_{\alpha}$ in a superfield treatment of super-Yang-Mills). The remaining fields are the corresponding antifields, in the Batalin-Vilkovisky (BV) sense. It is striking that one inevitably is lead to the BV formalism. It of course exists also in a component formalism, but when one uses pure spinors it is not optional. This means that any action formed in this formalism will be a BV action, and that the appropriate consistency relation (encoding the generalised gauge symmetry) is the master equation. 


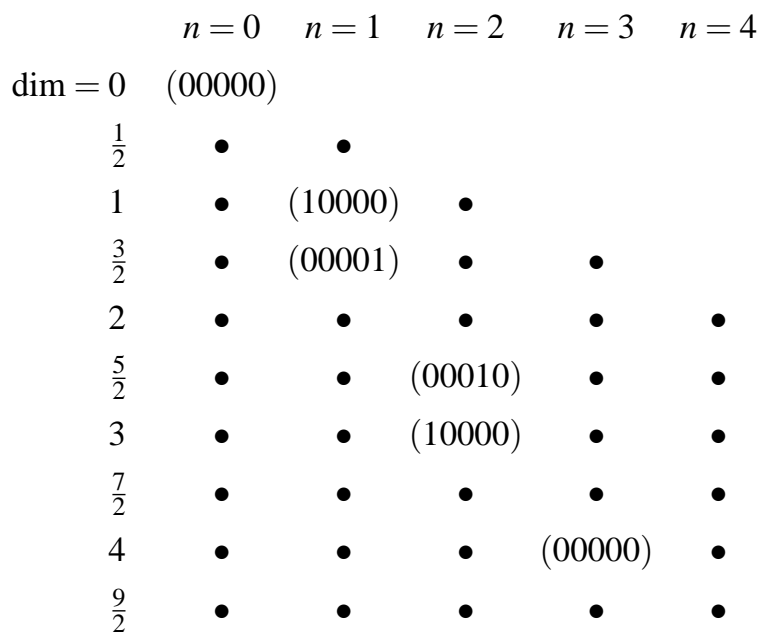

Table 1. The cohomology of the $D=10$ SYM complex.

To go from the zero-mode cohomology to the complete cohomology, one easily convinces oneself that component fields in the modules contained in the zero-mode cohomology will be subject to differential constraints in the modules of the zero-mode cohomology in the next column to the right. This gives the proper relations for the linearised on-shell super-Yang-Mills multiplet. (If a multiplet is an off-shell representation of supersymmetry, as is generically the case for half-maximal or lower supersymmetry, there will consequently be no anti-fields in the cohomology. These instead come in a separate pure spinor superfield [12].)

This far, we have not considered the actual solutions of pure spinor constraint, but rather regarded the pure spinor as a book-keeping device. When one wants to write down an action, this is no longer possible. For an action, a measure is needed. The linearised action should be " $\int \Psi Q \Psi$ " for some suitable definition of " $\int$ ". Clearly, " $\int$ " must have ghost number -3 . In the cohomology, there is a singlet at $\lambda^{3} \theta^{5}$. Defining a measure as a "residue", picking the corresponding component, has the right ghost number, and also the correct dimension. However, it is singular, so components of $\Psi$ with high enough power in $\lambda$ or $\theta$ drop out of the putative action defined in this manner, and the equation of motion $Q \Psi=0$ does not follow. Still, the corresponding tensorial structure can be used for an invariant integral over $\lambda$. It is clear from the partition function (3) that $\lambda$ contains 11 degrees of freedom (out of the 16 for an unconstrained spinor). Explicit solution of the pure spinor constraint also shows that when imposed on a complex spinor, only 5 out of the ten constraints are independent (see e.g. [5] for details). Defining the scalar at $\lambda^{3} \theta^{5}$ as $T_{\alpha_{1} \alpha_{2} \alpha_{3}}^{\beta_{1} \ldots \beta_{11}} \varepsilon_{\beta_{1} \ldots \beta_{16}} \lambda^{\alpha_{1}} \lambda^{\alpha_{2}} \lambda^{\alpha_{3}} \theta^{\beta_{12}} \theta^{\beta_{13}} \theta^{\beta_{14}} \theta^{\beta_{15}} \theta^{\beta_{16}}$, where $T$ thus is a Lorentz invariant tensor, one defines the conjugate invariant tensor $\tilde{T}^{\alpha_{1} \alpha_{2} \alpha_{3}} \beta_{1} \ldots \beta_{11}$, and the integration is

$$
[d \lambda] \lambda^{\alpha_{1}} \lambda^{\alpha_{2}} \lambda^{\alpha_{3}} \sim \tilde{T}_{\beta_{1} \ldots \beta_{11}}^{\alpha_{1} \alpha_{2} \alpha_{3}} d \lambda^{\alpha_{1}} \wedge \ldots \wedge d \lambda^{\alpha_{11}}
$$


In ref. [7], Berkovits solved the problem how to make sense of this integration and using it as part of a non-singular measure for the pure spinor superspace. The solution involves a non-minimal set of pure spinor variables, which in addition to $\lambda^{\alpha}$ contains a bosonic conjugate spinor $\bar{\lambda}_{\alpha}$ (which in Euclidean signature can be viewed as the complex conjugate of $\left.\lambda^{\alpha}\right)$ obeying $\left(\bar{\lambda} \gamma^{a} \bar{\lambda}\right)=0$ and a fermionic spinor $r_{\alpha}$ with $\left(\bar{\lambda} \gamma^{a} r\right)=0$. The new BRST operator is $Q=\lambda^{\alpha} D_{\alpha}+\frac{\partial}{\partial \bar{\lambda}_{\alpha}} r_{\alpha}$, and its cohomology is independent of $\bar{\lambda}$ and $r$. One assigns ghost number -1 and dimension $1 / 2$ to $\bar{\lambda}$ and ghost number 0 and dimension $1 / 2$ to $r$. The measure for $\bar{\lambda}$ is the complex conjugate to the one defined in Eq. (4) for $\lambda$, and for $r$ :

$$
[d r] \sim \star \tilde{T}^{\alpha_{1} \alpha_{2} \alpha_{3}}{ }_{\beta_{1} \ldots \beta_{11}} \bar{\lambda}_{\alpha_{1}} \bar{\lambda}_{\alpha_{2}} \bar{\lambda}_{\alpha_{3}} \frac{\partial}{\partial r_{\beta_{1}}} \ldots \frac{\partial}{\partial r_{\beta_{11}}} .
$$

Using these integration measures, and the ordinary ones for $x$ and $\theta$, we list the dimensions and ghost numbers for the theory after dimensional reduction to $D$ dimensions in Table 2. So, the ghost numbers match, and also the dimensions ( $\frac{1}{g^{2}}$ has dimension $D-4$ in $D$ dimensions).

\begin{tabular}{|r|r|r|}
\hline & gh\# & $\operatorname{dim}$ \\
\hline$d^{D} x$ & 0 & $-D$ \\
$d^{16} \theta$ & 0 & 8 \\
{$[d \lambda]$} & 8 & -4 \\
{$[d \bar{\lambda}]$} & -8 & 4 \\
{$[d r]$} & -3 & -4 \\
\hline total & -3 & $-(D-4)$ \\
\hline
\end{tabular}

Table 2. The dimensions and ghost numbers of the $D=10$ measure.

The $\lambda$ and $\bar{\lambda}$ integrations are non-compact and need regularisation. In ref. [7] this is achieved, following ref. [17], by the insertion of a factor $N=e^{\{Q, \chi\}}$. Since this differs from 1 by a $Q$-exact term, the regularisation is independent of the choice of the fermion $\chi$. The choice $\chi=-\bar{\lambda}_{\alpha} \theta^{\alpha}$ gives $N=e^{-\lambda^{\alpha} \bar{\lambda}_{\alpha}-r_{\alpha} \theta^{\alpha}}$ and regularises the bosonic integrations at infinity. At the same time, it explains how the term at $\theta^{5}$ is picked out, this follows after integration over $r . N$ has definite ghost number 0 for the assignments for ghost number and dimension above (although any other assignment gives the correct ghost number and dimension for the non- $Q$-exact part).

An action for ten-dimensional super-Yang-Mills (or any dimensional reduction) can now be written in the Chern-Simons-like form [5]

$$
S=\frac{1}{2 g^{2}} \int\left\langle\Psi, Q \Psi+\frac{1}{3}[\Psi, \Psi]\right\rangle_{\mathbf{a d j}} .
$$


Note that there is no 4-point coupling. The component field 4-point coupling arises after elimination of unphysical components. One must however remember that this is a classical BV action. It obeys the classical master equation $(S, S)=0$, where the antibracket takes the simple form

$$
(A, B)=\int A<\frac{\overleftarrow{\delta}}{\delta \Psi(Z)}[d Z] \frac{\vec{\delta}}{\delta \Psi(Z)}>_{\text {adj }} B
$$

In order to perform quantum calculations with path integral over $\Psi$, gauge fixing has to be implemented. This involves traditional gauge fixing (of the component gauge field) as well as elimination of the anti-fields. I will comment briefly on gauge fixing towards the end of the lecture.

The considerations so far were concerned with pure spinors in $D=10$, and the specific case of maximally supersymmetric Yang-Mills theory. Any maximally supersymmetric model (without self-dual tensor fields) may be given a similar treatment. The formulation of BLG and ABJM models were given in refs. [18],[19]. Also gravitational models have been considered [10],[13], although all that is known in such cases is that the linearised theory arises as pure spinor cohomology.

We would like to discuss operators acting on pure spinor superfields (much of this is due to Berkovits and Nekrasov [16],[20]). Due to the pure spinor constraint, derivatives with respect to $\lambda$ are not well defined. Only operators preserving the ideal generated by the pure spinor constraint are "gauge invariant". They must fulfil

$$
\left[\mathscr{O},\left(\lambda \gamma^{a} \lambda\right)\right]=0 \bmod \left(\lambda \gamma^{a} \lambda\right)
$$

Some such operators are straightforward to derive, among them are the number operator (related to ghost number) and Lorentz generator (its pure spinor part), $N=(\lambda w)$, $N^{a b}=\left(\lambda \gamma^{a b} w\right)$, where $w_{\alpha}=\frac{\partial}{\partial \lambda^{\alpha}}$. But there are more generators like this. In fact, it is possible to define a spinorial operator $u_{\alpha}$ that acts on the representations not present in the ideal exactly as $w_{\alpha}$ does, but vanishes on the ideal. For the ordinary pure spinors in $D=10$, this operator is

$$
u_{\alpha}=w_{\alpha}-\frac{1}{4(N+3)}\left(\gamma^{a} \lambda\right)_{\alpha}\left(w \gamma_{a} w\right) .
$$

The ordering in this expression is as it stands ( $u_{\alpha}$ acts from the left on $\Psi$ ), and the gauge invariance of $u_{\alpha}$ is due to a cancellation between single and double contraction, so it is genuinely quantum mechanical. It is straightforward to verify that $\left(u \gamma^{a} u\right)=0$ and $\left[u_{\alpha}, u_{\beta}\right]=0$. Any gauge invariant operator can be expressed in terms of $\lambda^{\alpha}$ and $u_{\alpha}$. It is worth noting that $(N+3) u_{\alpha}$ is polynomial, and that

$$
\left[u_{\alpha}, \lambda^{\alpha}\right]=\frac{11 N+48}{N+3},
$$

interpolating between 16 (the number of components in a full spinor) at $N=0$ and 11 (the number of components in a pure spinor) as $N \rightarrow \infty$.

Analogous gauge invariant "derivative operators" can be formed for other types of pure spinors. Take for example the pure spinors in $D=11$ relevant for supergravity. They 
fulfil $\left(\lambda \gamma^{a} \lambda\right)=0$, while $\left(\lambda \gamma^{a b} \lambda\right)$ and $\left(\lambda \gamma^{a b c d e} \lambda\right)$ remain unconstrained [10],[13],[14]. In this case, there are several irreducible representations at a given power of $\lambda$ which are outside the ideal. Due to the existence of two non-vanishing spinor bilinears, $u_{\alpha}$ is not uniquely defined by having ghost number -1 . If $u_{\alpha}$ is gauge invariant, one may also form e.g. $N^{a b}\left(\gamma_{a b} u\right)_{\alpha}$. The more complicated representation structure leads to a more complicated expression for $u_{\alpha}$. The simplest form possible is

$$
\begin{aligned}
u_{\alpha}= & w_{\alpha}-\frac{1}{16(N+5)}\left(\gamma^{a b} \lambda\right)_{\alpha}\left(w \gamma_{a b} w\right)+ \\
& +\frac{1}{8(N+5)(N+7)}\left[(N-13)\left(\gamma^{a} \lambda\right)_{\alpha}\left(w \gamma_{a} w\right)-\frac{1}{2}\left(\lambda \gamma^{a b} \lambda\right)\left(w \gamma_{a} w\right)\left(\gamma_{b} w\right)_{\alpha}-\right. \\
& \left.-\frac{1}{16}\left(\lambda \gamma^{a b} \lambda\right)\left(w \gamma^{c} w\right)\left(\gamma_{a b c} w\right)_{\alpha}+\frac{1}{192}\left(\lambda \gamma^{a b c d e} \lambda\right)\left(w \gamma_{a} w\right)\left(\gamma_{b c d e} w\right)_{\alpha}\right],
\end{aligned}
$$

while the other independent "derivative" also contains terms of order $\lambda^{3} w^{4}$.

The operators discussed this far use only the minimal pure spinor variables, and are strictly gauge invariant in the sense of Eq. (8). There are two ways of going beyond this. One is of course to use the non-minimal variables described above. The second pure spinor, $\bar{\lambda}$, has ghost number -1 . Another is to relax the condition of gauge invariance to allow BRST-trivial terms:

$$
\left[\mathscr{O},\left(\lambda \gamma^{a} \lambda\right)\right]=\left[Q, \mathscr{O}^{\prime}\right\} \bmod \left(\lambda \gamma^{a} \lambda\right) .
$$

This will not alter the invariance on physical fields in the cohomology.

Applications of operators with negative ghost number occur in a number of situations. One example is the condition that takes a non-maximally supersymmetric theory onshell. This amount to identifying a new pure spinor superfield whose lowest component is the auxiliary field, the vanishing of which implies the equations of motion. This was done in the minimal pure spinor framework for $D=6$ super-Yang-Mills theory in ref. [12]. The cohomology for that model is typical for a non-maximally supersymmetric theory, in that the antifields are absent. The cohomology is (with $D_{3} \oplus A_{1}$ notation for the Dynkin labels):

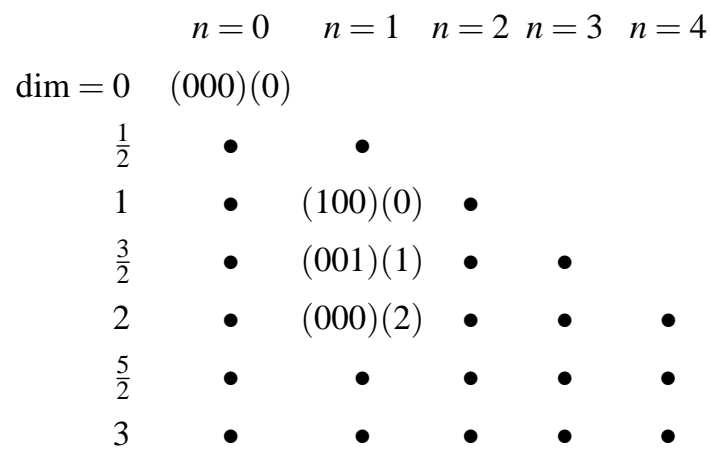


The antifields come in the current multiplet, sitting in a "conjugate field" in the auxiliary field representation $(000)(2)$ with cohomology

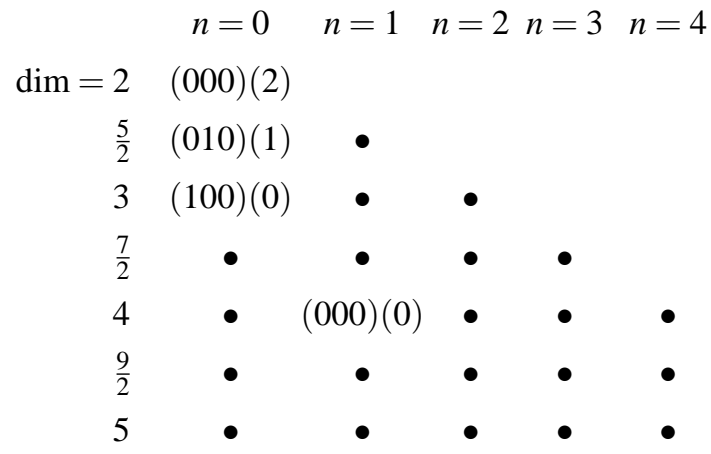

It is clear that the wanted operator has the schematic structure $w D^{3}$. A careful treatment shows that it can not be found using the strict gauge invariance of Eq. (8), but has to be gauge invariant only modulo $Q$-exact terms, as in Eq. (12). I refer to ref. [12] for the explicit form of the operator.

In the non-minimal formalism, there is a bigger choice of gauge invariant operators. The pure spinor $\bar{\lambda}$ has ghost number -1 , and an operator $(\lambda \bar{\lambda})^{-1} \bar{\lambda}_{\alpha}$ can be used instead of $w_{\alpha}$. Presumably, the construction of the operator above can be performed using nonminimal pure spinors. Such a formulation would be better suited for formulating an action.

Similar considerations become relevant when one tries to formulate an action for $D=11$ supergravity. There are two ways of obtaining the linearised multiplet. One may use a scalar field $\Psi$ of ghost number 3 and dimension -3, starting out with ghost for ghost for ghost for the 3-form tensor field. Or, one can use a vector $\Phi^{a}$ of ghost number 1 and dimension -1, starting with the diffeomorphism ghost. $\Phi^{a}$ has the additional invariance $\Phi^{a} \approx \Phi^{a}+\left(\lambda \gamma^{a} \rho\right)$ for any $\rho$. The ghost number 0 parts of these fields are the superspace 3 -form component with fermionic indices, and the lowest-dimensional component of the super-vielbein, respectively. We do not list the cohomologies here, but refer to ref. [10], where it was shown in that both these fields give the correct cohomology. The measure in this case has ghost number -7 , and a linearised action can be formed as $\int \Psi Q \Psi$. We would like to examine possible interaction terms. It is easy to see, from matching of ghost number and dimension as well as gauge invariance, that the only natural candidate for a three-point coupling constructed from $\Psi$ and $\Phi^{a}$ is

$$
\int\left(\lambda \gamma_{a b} \lambda\right) \Psi \Phi^{a} \Phi^{b}
$$

The insertion of $\left(\lambda \gamma_{a b} \lambda\right)$ ensures the gauge invariance under the "extra" symmetry $\Phi^{a} \approx \Phi^{a}+\left(\lambda \gamma^{a} \rho\right)$ through the Fierz identity $\left(\lambda \gamma_{a b} \lambda\right)\left(\gamma^{b} \lambda\right)_{\alpha}=0$, holding for pure spinors. However, we can not view the two fields as independent. There has to be a relation of the type $\Phi^{a}=R^{a} \Psi$, where $R^{a}$ is an operator with ghost number -2 and dimension 2. If $\left[Q, R^{a}\right]=0$, the master equation will be satisfied to this order. Given the experience from $D=6$ super-Yang-Mills, I find it likely that the best way to construct $R^{a}$ 
will be in the non-minimal formalism. The construction is under way. It is likely that this deformation of the linearised supergravity will generate the full interacting theory. How feasible calculations to higher orders are is unclear, and will depend on the algebraic properties of $R^{a}$. There is a striking similarity between the interaction term (13) and the Chern-Simons term $C \wedge H \wedge H$. The field $\Psi$ contains the connection $C$ while $\Phi^{a}$ only contains $H=d C$. The Chern-Simons term should be produced by Eq. (13).

Finally, a few words on gauge fixing. This is a further example of a situation where an operator of negative ghost number is needed. Gauge fixing in the Batalin-Vilkovisky formalism requires both elimination of the antifields and "ordinary" gauge fixing. In the pure spinor framework, the requirement is strong, since we do not want to break the field into different parts according to ghost number. Both tasks have to be fulfilled by a single condition $b \Psi=0$. The standard procedure (in superstring theory) is to take $b$ as the " $b$ ghost". Since reparametrisation invariance is not associated to a constraint in the pure spinor formalism, the $b$-ghost must be composite. It was constructed for the superstring in ref. [7] using non-minimal variables. For the super-Yang-Mills case (corresponding to second quantisation of a superparticle) it becomes simpler. It obeys $\{Q, b\}=\square$, which gives a good propagator. There are however problems associated with the singularity of $b$ at $\lambda=0$. I will not go into this problem here. A possible resolution was proposed in ref. [20].

\section{ACKNOWLEDGMENTS}

The author would like to thank Bengt E.W. Nilsson, Ulf Gran, Dimitrios Tsimpis, Pierre Vanhove, Nathan Berkovits, Niclas Wyllard and Pietro Antonio Grassi for discussions and comments.

\section{REFERENCES}

1. B.E.W. Nilsson, "Pure spinors as auxiliary fields in the ten-dimensional supersymmetric Yang-Mills theory", Class. Quantum Grav. 3 (1986) L41.

2. P.S. Howe, "Pure spinor lines in superspace and ten-dimensional supersymmetric theories", Phys. Lett. B258 (1991) 141.

3. P.S. Howe, "Pure spinors, function superspaces and supergravity theories in ten and eleven dimensions", Phys. Lett. B273 (1991) 90.

4. N. Berkovits, "Super-Poincaré covariant quantization of the superstring", J. High Energy Phys. 0004 (2000) 018 [arXiv:hep-th/0001035].

5. N. Berkovits, "Covariant quantization of the superparticle using pure spinors", J. High Energy Phys. 0109 (2001) 016 [arXiv:hep-th/0105050].

6. N. Berkovits, "ICTP lectures on covariant quantization of the superstring", proceedings of the ICTP Spring School on Superstrings and Related Matters, Trieste, Italy, 2002 [arXiv:hep-th/0209059.]

7. N. Berkovits, "Pure spinor formalism as an $\mathrm{N}=2$ topological string", J. High Energy Phys. 0510 (2005) 089 [arXiv:hep-th/0509120].

8. M. Cederwall, B.E.W. Nilsson and D. Tsimpis, "The structure of maximally supersymmetric superYang-Mills theory-constraining higher order corrections", J. High Energy Phys. 0106 (2001) 034 [arXiv:hep-th/0102009].

9. M. Cederwall, B.E.W. Nilsson and D. Tsimpis, "D=10 super-Yang-Mills at $O\left(\alpha^{\prime 2}\right)$ " , J. High Energy Phys. 0107 (2001) 042 [arXiv:hep-th/0104236]. 
10. M. Cederwall, B.E.W. Nilsson and D. Tsimpis, "Spinorial cohomology and maximally supersymmetric theories", J. High Energy Phys. 0202 (2002) 009 [arXiv:hep-th/0110069]; M. Cederwall, "Superspace methods in string theory, supergravity and gauge theory", Lectures at the XXXVII Winter School in Theoretical Physics "New Developments in Fundamental Interactions Theories", Karpacz, Poland, Feb. 6-15, 2001, arXiv:hep-th/0105176.

11. M. Movshev and A. Schwarz, "On maximally supersymmetric Yang-Mills theories", Nucl. Phys. B681 (2004) 324 [arXiv:hep-th/0311132].

12. M. Cederwall and B.E.W. Nilsson, "Pure spinors and $\mathrm{D}=6$ super-Yang-Mills", arXiv:0801.1428.

13. M. Cederwall, U. Gran, M. Nielsen and B.E.W. Nilsson, "Manifestly supersymmetric M-theory", $J$. High Energy Phys. 0010 (2000) 041 [arXiv:hep-th/0007035]; "Generalised 11-dimensional supergravity", arXiv:hep-th/0010042.

14. M. Cederwall, U. Gran, B.E.W. Nilsson and D. Tsimpis, "Supersymmetric corrections to elevendimensional supergravity", J. High Energy Phys. 0505 (2005) 052 [arXiv:hep-th/0409107].

15. P.S. Howe and D. Tsimpis, "On higher order corrections in M theory", J. High Energy Phys. 0309 (2003) 038 [arXiv:hep-th/0305129].

16. N. Berkovits and N. Nekrasov, "The character of pure spinors", Lett. Math. Phys. 74 (2005) 75 [arXiv:hep-th/0503075].

17. R. Marnelius and M. Ögren, "Symmetric inner products for physical states in BRST quantization", Nucl. Phys. B351 (1991) 474.

18. M. Cederwall, " $\mathrm{N}=8$ superfield formulation of the Bagger-Lambert-Gustavsson model", J. High Energy Phys. 0809 (2008) 116 [arXiv:0808.3242].

19. M. Cederwall, "Superfield actions for $\mathrm{N}=8$ and $\mathrm{N}=6$ conformal theories in three dimensions", $J$. High Energy Phys. 0810 (2008) 070 [arXiv:0809.0318].

20. N. Berkovits and N. Nekrasov, "Multiloop superstring amplitudes from non-minimal pure spinor formalism", J. High Energy Phys. 0612 (2006) 029 [arXiv:hep-th/0609012]. 